\title{
The Personality of the Influencers, the Characteristics of Qualitative Discussions and Their Analysis for Recommendations to Cultural Institutions
}

\author{
Vassilis Poulopoulos $1, *,+(\mathbb{D}$, Costas Vassilakis $2,+(\mathbb{D})$, Angeliki Antoniou $2,+(\mathbb{C}$, \\ George Lepouras ${ }^{2,+}\left(\mathbb{D}\right.$, Anastasios Theodoropoulos ${ }^{2}(\mathbb{D})$ and Manolis Wallace ${ }^{1,+}$ \\ 1 ГАВ LAB - Knowledge and Uncertainty Research Laboratory, University of the Peloponnese, 22131 Tripolis, \\ Greece; wallace@uop.gr \\ 2 Deparment of Informatics and Telecommunications, University of the Peloponnese, 22131 Tripolis, Greece; \\ costas@uop.gr (C.V.); angelant@uop.gr (A.A.); gl@uop.gr (G.L.); theodor@uop.gr (A.T.) \\ * Correspondence: vacilos@uop.gr; Tel.: +30-697-2700-533 \\ + These authors contributed equally to this work.
}

Received: 23 August 2018; Accepted: 5 October 2018; Published: 11 October 2018

\begin{abstract}
Social media usage is affecting peoples' views through opinion sharing, a fact that has started to attract cultural institutions, as it is possible that this procedure can possibly be a part of a museum experience. As the main goal of a cultural institution is the maximization of senses stimulation, the device that is offered to the visitors' hands everyday and every moment, becomes an important tool for the art spaces. In this notion we perform research on issues that can be of great importance for the museum's online presence and attraction. We focus on establishing the personality of influencers related to culture, as well as the characteristics of qualitative discussions on the social media. Crosscult Project is an EU funded project, that aims to spur a change in the way European citizens appraise History, and sets that basis of our research as the experiments are conducted within its scope of. Through the experimental procedure, we collect information in order to define the character of the influencer and the substances of a "serious" conversation. "Serious" conversations are regarded the ones in which a cultural organization can participate actively and benefit from the participation. We present the results of our experimental evaluation and analyze how cultural institutions can benefit from the outcomes of our research.
\end{abstract}

Keywords: personalization; user modeling; personality traits; social media influencers; cultural informatics; serious conversations; digital humanities

\section{Introduction}

Contemporary museums carefully select objects, plan exhibition themes and layouts, design accompanying information and create a certain museum ambiance, all to ensure that visitors are engaged and appreciate the overall quality of the museum experience [1]. The main goal is to stimulate the senses and to activate different cognitive, conative and affective processes, like high order thinking and reflection, desire to know, emotional arousal, etc. [2-6]. In addition, nowadays the cultural institutions are able to access and use a number of technological tools to assist them in their endeavors. The visitor experience can be supported before, during and after the visit using different technological solutions spanning from museum web pages, to mobile devices and social media [1,7-9]. Most of the aforementioned applications require active participation and involvement of the visitors. In parallel, an increasing number of cultural venues support innovative procedures, during the visits, in order to offer an enhanced experience to their audience. What can really affect the way cultural institutions act, is a further analysis of a procedure that can be initiated during the visit and continue 
as a post-visit interaction; the latter not necessarily including only visitors but a broader audience of people willing to interact with the venue. In other words, the cultural institution can benefit from visitors that can act as ambassadors of the stimulation of feelings and the sharing of the visit experience.

In our everyday life, some people can affect us more than others, while there are a number of persons that can possibly impact numerous other people easily through their opinion (the so called influencers). Influencers is a trend term, the usage of which arouse with the extensive use of social media. The marketing sector puts great effort in studying how the personality of people affects their influential role [10]. In parallel, the extended use of social media leads to studies concentrating on opinion leading within their scope [11]; cultural experiences are not excluded. In order to understand the extent of the "influencing trend", a number of people search for information about places, events and advice from people that are believed to be "experts" or individuals with dominant personalities. The communication of messages through influencers is generally considered as a good practice to maximize the experience, if people follow their advice [12]. On this basis, managing to recognize an influencer, as either an expert of a field or an opinion leader, introduces a strong possibility to understand how others can be affected $[13,14]$.

Influencing personalities can manipulate others in various ways; in particular-and in relation to cultural heritage- they can possibly motivate in visiting a place, generate trends, create groups of people that can promote engagement as well as start discussions. The actions of the influencers are important for cultural institutions for two reasons. Firstly the influencers can take part and play an important role for spreading messages on behalf of the cultural institution and secondly, the cultural spaces can possibly learn from the influencers' behavior and act accordingly. Furthermore, as the existence of a cultural space relies on the spread of the "correct" message, any misunderstanding or dissemination of the wrong words can mislead the audience and have a negative effect on the cultural institution. The aforementioned issues lead to the assumption that it is important, on the one hand, to recognize the visitors' - or potential visitors' - personality and, on the other hand, to be able to track influencing or relevant conversations. In our research, we put the focus on the personality of people that are able to easily motivate and manipulate others, by trying to establish the personality of the so-called influencers. Furthermore, we analyze their impact on others, and how this impact is reflected on discussions, and uncover the aspects of such discussions. We refer to serious conversations as "important" conversations, as they are places for active involvement of a cultural organization in order to gain more audience, spread their message and achieve higher rate of impact. The research performed is done within the scope of "CrossCult" EU Project, and is performed through the pilot procedures. The moto of CrossCult project is "Empowering reuse of digital cultural heritage in context-aware crosscuts of European history" and it aims to spur a change in the way European citizens appraise History. In the scope of the reported research, the procedure includes construction of ad-hoc social networks and measurement of interactions of the participants in real time during and after their visit to predefined cultural spaces. One aspect of the pilot execution-expressed by the current research-is the examination of the personality of the influencing visitors, the attributes that transform a simple online conversation to an important discussion as well as how all the aforementioned can be beneficiary for the cultural spaces.

The rest of the paper is structured as follows. The next section presents the related work that is done in relevant research areas and Section 3 presents in detail our research methodology. Section 4 presents our experimental procedures and the next section presents a discussion on the founding. We conclude with future work and remarks.

\section{Related Work}

The term influencer is not something novel, it arouse as a term that is extensively used in social media. According to Freberg et al. [15], it refers to people (or just online personas) whose attitude, way of expressing opinions, and viewpoints can massively affect other people. As a research assumption, the previous can be performed intentionally, can be related to the position the society, 
culture or work, or it can even be a result of continuous media presentation [16]. As the variety of social media is large we examine the influencers as they act in social media like Facebook (https: //www.facebook.com-Facebook), Twitter (https:/ /www.twitter.com-Twitter) or Google+ (https: // plus.google.com-Google Plus). Common influencers could be people like athletes, rock stars, actors, models, politicians, mentors, etc., however, research shows that everyone could be an influencer under certain circumstances [16]. Peng and Sun [17] state that influencers affect the growth of social media conversations by not pretending to be a major source of information or news but they comment as event happen. Individual social media influencers seem to have their own unique style, or their social media "voice", when they express their opinion and interact with others [18].

From a technical point of view, recognition of influencing personalities in social media includes measuring parameters related to the peoples' number of posts, likes, comments and reproduction of their content by others, analyzing quality aspects of the comments and finally estimate the number of people that received the "message", the so-called "reach". Several different research paths focused on establishing influencing personalities in social media. Peng and Sun [17] applied a dynamic diffusion model, in order to find influencers in discussions, and to predict others from different topics, but within a specific time frame. Finding influencers and serious conversations is extremelly important for marketing purposes as well, which is the reason research is done in the field, focusing on use of dedicated algorithms that can spot influencers for a particular product [19]. It is also important how others recognize, receive and react to the actions of the influencers, a procedure that is part of the research from Freberg et al. [15], who develop a technique that identifies attributes of social media influencers and try to help organizations improve the social media skills by applying the deriving attributes.

In parallel to analyzing the personality of visitors of a cultural venue, and extracting information about their personality, an algorithmic procedure is also applied in order to discover the serious conversations. Algorithms have been also used successfully in the past to analyze social media conversations [20]. Measuring the number of comments, length of text, reactions to original text and comments, number of people that participate in the conversation and definition of the adjectives in the text, is some of the key metrics that can help us recognize qualitative discussions. Analysis of the adjectives plays an important role in natural language processing and their role in a text is part of NLP research in several fields [21-23]. Furthermore, by analyzing the factors that make a discussion important we should be able to re-create similar discussions, or even participate in an automated way (e.g., bots). Moreover, among the primary scopes of a cultural venue is the maximization of visitor experience. As the use of social media and online content can affect the attitude of a visitor (or a future visitor), it is crucial for the venues to be able to participate, publish and be aware of serious conversations; the people that can possibly generate and participate in them should also be researched. The scope of "serious conversations" recognition is thus twofold. At first, we are able to alert the cultural institution about the existence of such a conversation, as it is a place of interaction with the public. Secondly, by recognizing the characteristics of conversations, the cultural institution is able to initiate such kind of conversations in order to attract the attention and raise the awareness of its audience, thus increasing its social media influencer capital [15] and improving the venue performance [24].

Despite the fact that analysis of social media influencers is a research path with very high volume, there seems to be little research in relation to cultural heritage. A gap in the use of technology and communication between high and popular culture was revealed in a study back in 2012 [25]. The comparison reveals a significant difference in social media reach between popular and high culture, with the latter being extremely poor in new technology adoption. High culture is not only a matter of the exhibit itself but it can be directly related to the narrative generated and the targeted audience. From the aforementioned it is obvious that high culture requires a lot more technological attention.

Therefore, the current work focuses on:

- identification of the type of personality of the Cultural Influencer. 
- identification of the characteristics of the Cultural Influencing discussion.

Apart from the aforementioned, this work will furthermore focus in empowering the cultural institutions in order to have a more effective presence in serious conversations, generate trends, identify influencers and engage visitors, points that make this research innovative.

\section{Research Methodology}

According to the research project's pilot, a group of people visited the Archaeological Museum of Tripolis (Greece) and they interacted to each other during and after their visit. During the visit their interactions and conversations were recorded by the organizers while, after visiting the museum, the participants were asked to participate in a discussion on an online Google document shared on Google Drive (https://drive.google.com-Drive by Google). Participants are provided with 3 discussion topics, relevant to their visit, to chose from:

- Discussion topic 1: "Nudity in antiquity and today". The experience in the museum included instructions related to the symbolism of the naked body in ancientness. There were 4 participants ( 2 women, 2 men) that had previously met and briefly introduced.

- Discussion topic 2: "Appearance as a comedy element in Classic Greek cinema". This topic was relevant to the museum information they had accessed about appearance of statues, that functions as a code for the archaeologists, in order to understand what this artwork was. There were 6 participants ( 3 women and 3 men), that had previously met and briefly introduced.

- Discussion topic 3: "Comparing images". Participants had to compare two images of women from Sicily. The first images showed young Romans wearing bikinis and playing a ball game. This was a mosaic from Villa Romana del Casale (4c AD). The other image, showed contemporary nuns in Sicily. There were 3 participants in this discussion, all women, good friends since before the experiment commenced.

In addition, there were two more ongoing discussions on Facebook, from which it was possible to extract a broader number of implicit interactions (e.g., likes, shares, etc.):

- Discussion topic 4: "Comparing images". The same images as in discussion topic 3 were used. In this discussion there were 11 participants ( 5 women, 6 men). Most people did not know each other (only 3 women knew each other since before). The conversation is presented in Figure 1.

- Discussion topic 5: "Comparing images". Images of ex votos were used from Christian contemporary churches (Catholic, Orthodox) and ancient ones from archaeological museums. In this discussion, there were 10 participants ( 3 women, 7 men). Most participants knew each other since before.

The topics to be discussed are not randomly selected, or just related to the museum content, but were proposed by two experts in the field of humanities, specializing in Social and Clinical Psychology, and History and Archeology. The content was such that it could be possible that the participants would have totally different attitude on the same subject, and that they would have to support their opinion with strong arguments, trying to find supporters and rivals. The time of the conversations was limited to one week, after which, the participants were asked to conduct a personality test. Despite the fact that the conversation, especially in social media, can last for a long time, the data was collected and concern only the time limit provided. The purpose of the personality test was to manage the manual extraction of each participant's profile. 

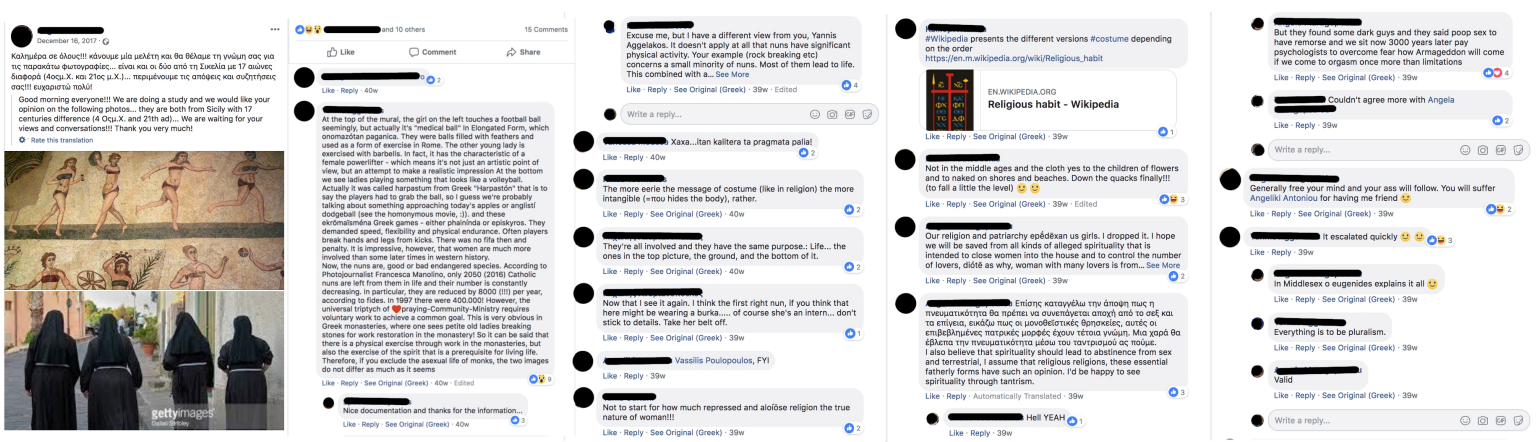

Figure 1. Facebook Conversations.

In fact, as the scope of our research is twofold, we engage a number of different research methodologies to extract enough information, so as to safely decide on the personality of the influencers and locate the "ingredients" of the serious discussions. To achieve the aforementioned, we perform a set of analyses on the participants, the data deriving from social media and their combination. As depicted in Figure 2, starting from the analysis on social media content-recorded by the participants-to locate the influencers, we progress to the DiSC personality test so that we are able to recognize the personality of the influencers and we perform an analysis of bonds between the participants. Next, we can locate serious conversations and proceed to NLP analysis and Reflection analysis in order to find the aspects of the serious conversations.

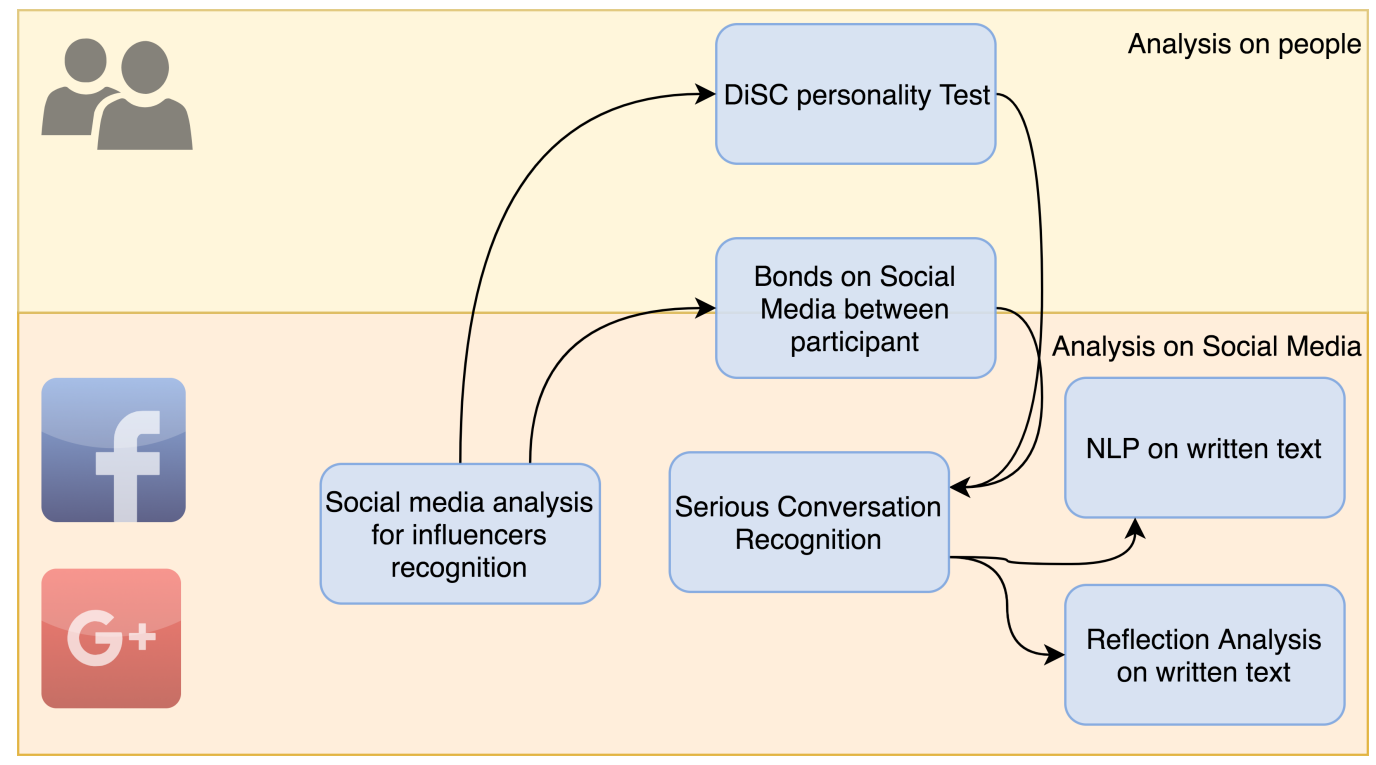

Figure 2. Different stages of analysis.

\subsection{Personality Test}

The data collected from the online conversations and the visits at the museums are enough to have a first approach to each participant's personality. Nevertheless, it is important to have precise knowledge of the temperament of each participant in order to extract one of the main aspects of our research work, the personality of the influencers. In our case we used the DiSC personality test [26], in order to get information about each person. The current personality test, as its name implies, identifies 4 main dimensions:

- Dominance: The way people deal with problems and control situations.

- Influence: The way people deal with other people, communicate and relate to others. 
- Steadiness: The temperament people have, like patience, persistence and thoughtfulness.

- Compliance: The way people approach and organize their activity, procedures and responsibility.

With the current personality classification it is possible to locate relations between people themselves, and between people and tasks, leading to improvements in work productivity, as well as teamwork and development of communication skills [27-29]. In parallel, research is done using the same personality test in order to make prediction about group format, member's satisfaction and performance of tasks [30]. A sample result of the DiSC personality test is depicted in Figure 3.

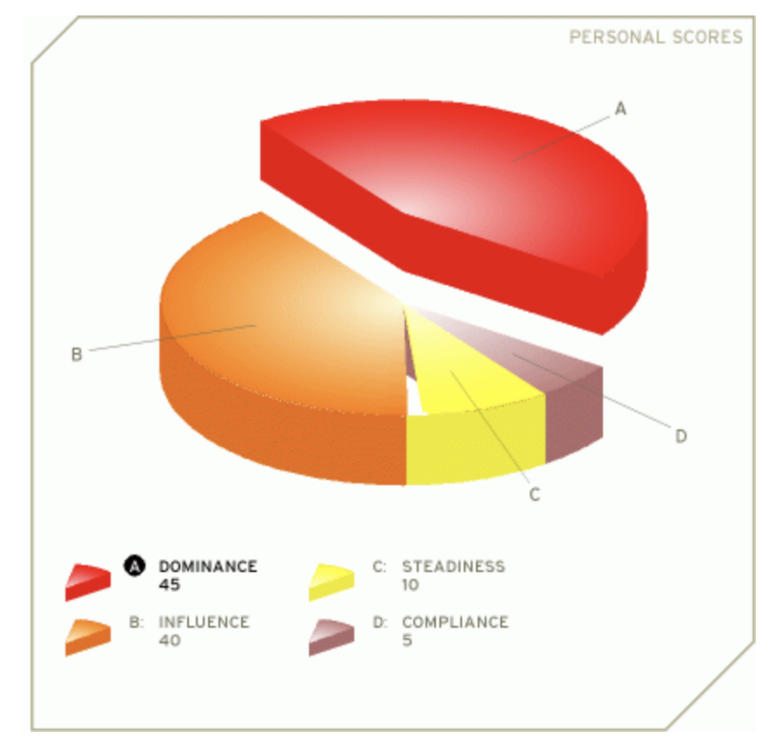

Figure 3. DISC personality sample result.

\subsection{Social Media Analysis}

While the personality test is able to provide information about the characteristics of each different user, and help us recognize the people that act as influencers, the analysis of social media provides metrics that can be used in order to realize the quantitative factors that can play role in an online conversation. In the case of Facebook the parameters identified as crucial in order to export information about an "influencing" discussion is related to:

- Number of reactions, with the side-note that likes are not equally calculated to other types of available reactions (emojis). These are calculated for the original starting post and for each comment. Likes and emojis are measured since past research has shown their importance in the perceived value of a discussion [31,32].

- Number of comments to original post, since research has indicated the importance of measuring comments to posts [33].

- Number of comments to comments [34].

- Number of people commented $[35,36]$.

- Number of people reacted [37].

All the aforementioned information needs to be determined in order to be able to recognize initially the types of conversations perceived as interesting for the cultural institutions, and secondly, understand the specific elements that create such a conversation. The social media analysis provided primarily quantitative data, whereas qualitative data derived for the natural language analysis described below. 


\subsection{Natural Language Analysis}

The present study also wished to study the qualitative attributes of cultural conversations. Natural language processing was performed to the conversations in order to extract information related to:

- Length of text, number of words and size of sentences.

- Parts of speech.

- Formality of language.

- Entities in the text.

- Sentiment of the text.

Our initial goal is to make interconnections between the results of the NLP as parts of the ingredients of the serious conversations. Natural language analysis allowed the first level of qualitative data gathering. In order to proceed with the natural language processing of the amount of texts produced by the experimental procedure we utilized Google Cloud NLP Service (https: / cloud.google. com/natural-language/ Google Cloud Natural Language).

However, research has proved that meaningful cultural discussions are also connected to reflections of social and historical phenomena, and re-interpretations of past and present [30,38-40]. In this light, reflection analysis was also performed.

\subsection{Reflection Analysis}

Reflections are the emotions that arouse by reading a text, and as such information regarding the number and type of reflections are collected. In order to perform such kind of analysis the taxonomy proposed by Soren [41] was used. The current taxonomy is used to describe cognitive alterations when facing cultural related content. In particular the visitors establish different ways to describe the alterations:

- novel understanding.

- idea of infinity/lasting phenomena.

- experiences with original objects.

- highly emotional experiences.

- unexpected senses.

- possibility to become motived.

Apart from the Soren taxonomy, extensive use of the Bloom taxonomy [42] in its revised form was done [43]. This can be done as the reflection can be regarded partially as a learning process [44]. The current taxonomy is formulated as a six level cognitive process including understanding/comprehension, application of knowledge, memory, creativity, analysis and evaluation. Using a combination of the two taxonomies on the collected data helped us reveal the reflections that can be present in texts related to cultural heritage.

\section{Experimental Evaluation}

The experimental evaluation has as main goal to reveal the personality of the influencing personalities in cultural related conversations, and secondarily to analyze the "ingredients" of a high level serious conversation with cultural related subject. An in-depth analysis of the results can lead to provision evidence to cultural spaces, in order to empower their presence and activities in social media. The procedure starts with a programmed visit at the Archaeological Museum of Tripolis, as part of a pilot procedure of "CrossCult" EU project, followed by online joint conversations, including all the visitors and their online friends. We furthermore examine each participants personality, both from the people that visited the museum, as well as the ones that just participated to the public online conversation in order to recognize their personality according to DiSC. 


\subsection{Personality of the Influencer}

After identifying the influencing participants we proceed with examining their personality according to the personality test. The main input for recognition of influencers were the online conversations, as well as the discussions and reactions during the live visit in the museum. In particular, an influencer is a person that creates a block of text that collects a number of reactions within the public space of the social media. Large number of reactions (e.g., likes or emojis), sharing of the opinion, text statements that express agreement, high post reach are just some of the metrics collected in order to find the texts that seem to be generated by potential influencers. In addition, apart from locating texts with high affection to other, we performed the DiSC personality test to the participants who accepted to take part in the personality assessment. Table 1 presents the different personalities identified by execution of the test.

Table 1. Personalities of participants.

\begin{tabular}{ccccc}
\hline Personality Trait & Dominant & Influencing & Steady & Compliant \\
\hline Number of People & 5 & 10 & 7 & 2 \\
\hline
\end{tabular}

The DiSC personality test does not present an absolute answer as for the person's personality but a relative approach depicted as a percentage towards each of the 4 different personality traits. An important observation was that in 4 of the cases people were equally influencing and dominant ( $>38 \%$ for each of the characteristics), and in 1 case a person was both steady and dominant $(>40 \%$ for each of the characteristics).

Another important part of the influencing participants identification, is monitoring the "physical" bonds that may exist between two online users. For this purpose the algorithm described by Bakshy et al. [45] was used. According to this algorithm, it is expected that a user responds better within an online conversation when a conversation is initiated by someone strongly connected with the user. The connection between user $i$ and user $j$ is expressed with the following:

$$
W_{i, j}=\frac{C_{i, j}}{C_{i}}
$$

where $C_{i}$ is the number of posts generated by user $i$ within the social network, and $C_{i, j}$ represents the number of posts generated by user $i$ within the same time period and are targeted or related to user user $j$. The target or relation can be found by analyzing tagging or posts that are recorded to the other users wall, timeline or posts.

Finding a strong bond between the participants is directly translated to locating influencing people among the participants before performing any other means of analysis. According to the generated results in at least one occasion the reaction of a specific user towards another participant was due to their strong bond of friendship. In our analysis, this type of affection between the users is still extremely useful for the cultural spaces, especially in the situations that social media can be used to empower engagement.

According to the aforementioned algorithm, there are three different types of interactions between two users on the Facebook. We distinguish these types as:

- Type A. User 1 posts directly to the "wall" of user 2.

- $\quad$ Type B. User 1 posts as reply to a post of user 2.

- Type C. User 1 posts as reply to a thread in which user 2 participates.

For the current algorithm it is only a matter of interaction and each type is considered equally to the end result. Table 2 presents the bonds calculated between user A.A. and other three participants with initials Y.A., I.B., K.K. and M.A. The user A.A. has published a total of 365 posts within the 
period of time of 18 months. The table presents how many of these posts were related to each of the user within the same period of time, and a high bond measure reveals a pre-existing relationship of influencer and influencee.

Table 2. Bonds between User A.A. and other four participants.

\begin{tabular}{ccccc}
\hline User & Type A & Type B & Type C & W (Bond Measure) \\
\hline User Y.A. & 6 & 6 & 0 & $12 / 365=3 \%$ \\
User I.B. & 8 & 13 & 19 & $40 / 365=11 \%$ \\
User K.K. & 3 & 4 & 0 & $7 / 365=2 \%$ \\
User M.A. & 5 & 4 & 7 & $16 / 365=4 \%$ \\
\hline
\end{tabular}

According to the results presented in Table 2 there is a strong connection between users A.A. and I.B. while checking the reverse connection as well proves who acts as the influencer and who is the influencee in the relation.

At this stage of the procedure, we should be aware of the participants, the connections that exist between them, the conversations and the attitude towards each other, as well as their personality. Furthermore, we have knowledge of comments that act as influencing text and the related personality. Figure 4 presents some of the texts recorded that include characteristics that make them be tagged as influencial. Apparently, it is obvious to assume that people that wrote influential texts can be considered as influencers. Consequently, a connection between the influencing users and their personality will lead us to the connection between influencing personalities and the extracted personality according to the DiSC assessment.
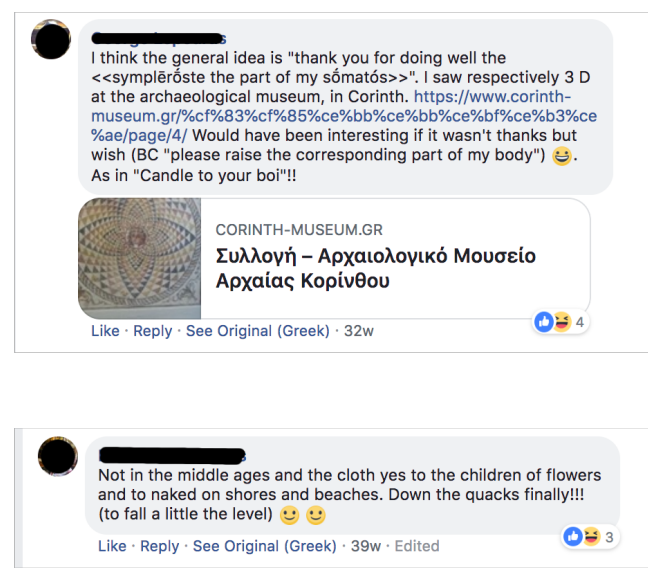

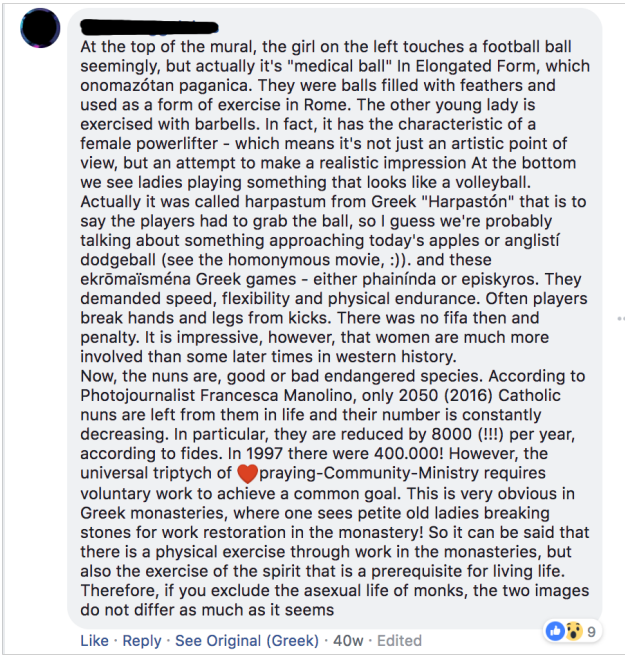

Figure 4. Influencing texts.

According to Table 3, there is a connection between personality traits and serious conversations. From the recorded results, it is obvious that the combination of influencing and dominant personalities led to influencing conversations. Other occasions in which a person is related to a single personality or in the case of the steady and compliant personality there was no obvious or direct connection with serious conversations.

The analysis proved that it is not only for people with "influencing" personality trait that compose influencing and serious conversations, but it seems more typical that the kind of personality that is superior when it comes to influencing conversation are people that combine Influence and Dominance personality traits. This partially proves the assumption of what is defined as influencer. It could be related to people with strong personality that have an accordingly higher social or work position as 
well as people that manage to manipulate others with their temperament and personality, or just a mixture of the above.

Table 3. Number of important texts based on personality.

\begin{tabular}{ccc}
\hline Personality & \# of Important Texts & \# of People \\
\hline Influencing only & 3 & 2 \\
Dominant only & 2 & 2 \\
Steady only & 2 & 2 \\
Compliant only & - & - \\
Influencing \& Dominant & 12 & 4 \\
Steady \& Compliant & - & - \\
\hline
\end{tabular}

\subsection{Characteristics of Influencing Conversation}

Another important aspect of our research is the recognition of the characteristics of the influencing conversation. In fact we need to understand the substances that make a conversation interesting for the cultural institutions. First of all we need to distinguish the serious conversations from the ones that do not seem to be "interesting" for a cultural institution.

If we consider the conversation to be a tree of text blocks, each branch depicting a comment with its sub-comments we recognize 4 paths in the conversations conducted to be interesting. Figure 5, presents the way a conversation tree can be formed. We furthermore highlight the nodes on the tree that have significant numbers of reactions, and highlight the path that is considered to include an "interesting" conversation.

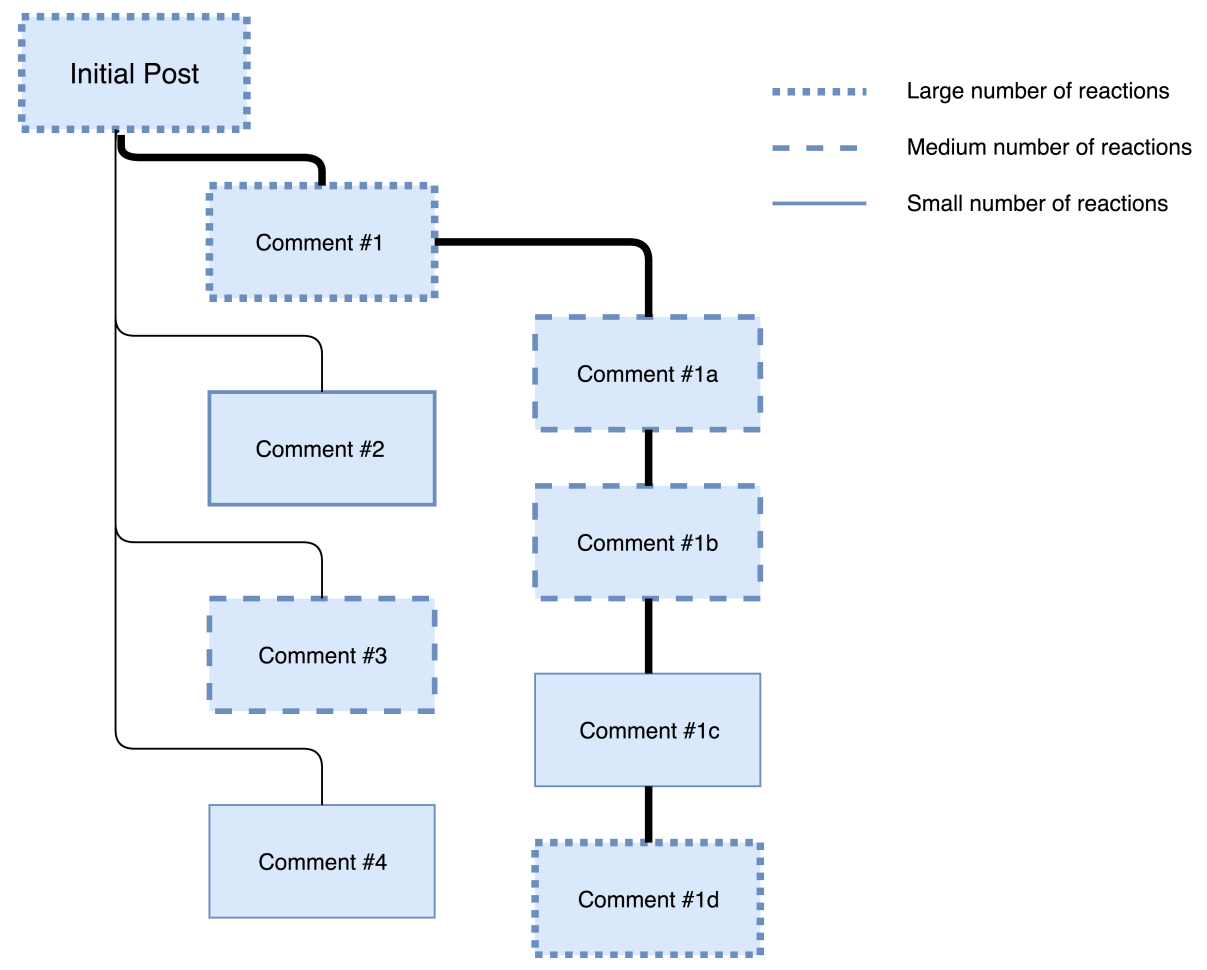

Figure 5. Conversation Tree.

In the conversation path we have used dotted line to highlight the texts with large number of reactions, dashed line was used for the conversations with medium reactions, and straight normal line was used for the conversations with small number of reactions. 
For these texts we identify patterns based on length of text and different parts of speech. In most of the cases a conversation starts with a large text (in the social media context), but does not necessarily contain equally large answers. An interesting observation is that many texts in serious conversations include emoticons/emojis, or external links (Figure 6).

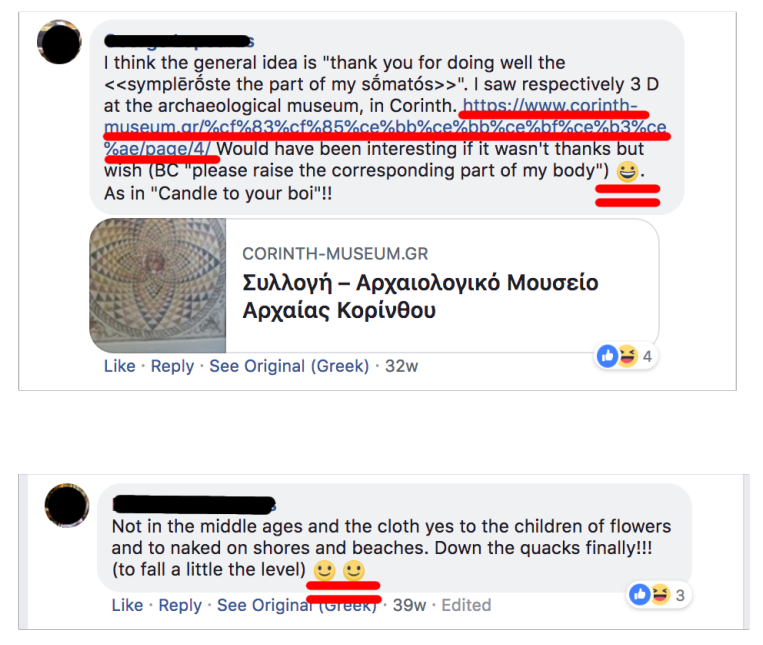

Figure 6. Influencing texts.

One of the parts of our research procedure was to extract the "ingredients" of a serious conversation on the social media having cultural context. In this scope, we perform linguistic analysis. The scope is to search if there is any specific pattern on the text that is related to parts of speech. According to analysis presented in Table 4, which presents the linguistic analysis of the serious conversation depicted in Figure 5, specific patterns were not identified. Instead, what was observed was that comments contain single, few or answers without words, like emoticons/emojis, moving gifs, external links or images. In addition, social media conversations do not always follow correct grammar rules. As such, it was difficult to identify any patterns in user inputs based on grammatical structure.

Table 4 depicts an example of a conversation analysis and what was observed based on Natural Language Processing.

Table 4. Natural Language Analysis of a Qualitative Conversation.

\begin{tabular}{cccccc}
\hline Text & Length (Words) & Adjectives & Nouns & Verbs & Sentiment \\
\hline Initial & 40 & $8(20 \%)$ & $12(30 \%)$ & $4(10 \%)$ & Neutral \\
Comment 1 & 340 & $35(10 \%)$ & $93(27 \%)$ & $60(17 \%)$ & Neutral \\
Comment 1a & 204 & $23(10 \%)$ & $53(24 \%)$ & $23(10 \%)$ & +0.12 \\
Comment 1b & 26 & $1(1 \%)$ & $12(46 \%)$ & $3(11 \%)$ & -0.09 \\
Comment 1c & 9 & $0(0 \%)$ & $2(22 \%)$ & $1(11 \%)$ & Neutral \\
Comment 1d & 23 & $4(17 \%)$ & $6(26 \%)$ & $4(17 \%)$ & -0.04 \\
\hline
\end{tabular}

Our research did not reveal any obvious pattern with the quantitative measurement of data. Qualitative analysis was then employed to look deeper into the data set. Each of the texts was analyzed towards their reflections; and an interesting pattern was observed. It was obvious that the "branches" of the serious conversations had significantly larger number of reflections compared to conversations that attracted less interest. Bloom and Soren taxonomies were utilized in order to find reflections into the conversation branches.

By analyzing the complete collection of texts according to the conversation tree already described and by locating the reflections of each conversation branch on the tree we clearly observed a pattern 
that associates directly a qualitative cultural related conversation to reflections. Table 5 displays the amount of reflections and their relations to serious and not interesting conversation. From the result, it is obvious that, the more the reflections into a conversation branch the more serious the conversation tends to be, as long as the cultural context is involved.

Table 5. Number of reflections per conversation branch.

\begin{tabular}{cc}
\hline Conversation & Number of Reflections (Total in Branch) \\
\hline Serious & more than 10 \\
Other & less than 3 \\
\hline
\end{tabular}

\section{Discussion}

The procedure, in which we focused on examining the personality of the influencers and the characteristics of qualitative conversations, revealed a number of important assumptions that make the research compelling. At first, it was observed that leading personalities having high levels of influence or dominance, as personality traits, are not by contract influencing individuals. It actually comes to the assumption that it is more like a mixture of the two types that can lead to influencing individuals. Nevertheless, the data derived from participants generating only from Greece, meaning that the results can be biased; on the other side the current work focuses more on the qualitative analysis, and as such, it is expected that the work will be done with a limited sample size. The latter implies that both the number of participants, as well as their origin, must be expanded in order to confirm the research outcomes in a broader audience. As such, part of the future work should focus on this path.

Furthermore, an important assumption that was made, is that in discussions within cultural context, especially when speaking about high culture, prior information on the subject leads to better results in the influencing procedure. In such conversations, even people that have high affection to others, can easily become followers rather than leaders, when they do not have any knowledge on the issue. It is also very usual for influencers in the cultural context to be absent when conversations tend to follow paths out of their knowledge. On the other hand, it is equally usual that influencing individuals tend to write having in mind that, what matters, is not their actual knowledge on the issue, but what all the others (usually the followers) believe is their knowledge. In this light, it is obvious that the mixture of Dominant and Influencing personalities have precedence in creating the necessary environment for an influential cultural discussion.

Moreover, we examined for patterns of serious, cultural related discussions in social media. It seems that social media use also implies an altered form of language used by participants. In this light, participants do not seem to use correct grammatical and syntactic structures when they express their opinions and feelings. As it was observed in the present work, adjectives, that are considered important for sentiment analysis, were used very scarcely, if not at all, in social media cultural discussions. People produced small texts and used few words if any. Sometimes, people expressed themselves with emojis. Therefore, social media qualitative analysis requires new forms of qualitative research that does not only focus on traditional written language, but it also includes new ways of expression (use of icons and symbols).

Finally, Reflection analysis was proven very useful for the analysis of cultural discussions on social media. Reflection is not only important for the processes inside the venues and during the cultural visits, but it also seems important during users' interaction with cultural content and each other on social media. Our research revealed that discussions that triggered high-level cognitive processes were the ones that received most user attention (comments and other reactions). In this light, it is important for cultural institutions that wish to have an active role in social media to create texts of reflective potential. 


\section{Conclusions and Future Work}

In this work we presented the research procedures and experimental evaluation that focused on examining the personality of the influencer of cultural related online conversation, and we also examined the characteristics of a serious conversation. As already mentioned, it is possible that due to the number of participants, and a possible cultural bias may have affected the outcomes. The future work will focus in further analysis of discussions having cultural context from a broader international audience. In this light, more modern and detailed personality trait tests can be conducted such as Big-5 [46]. Another experiment planned to be done as future work, is to manipulate discussions by inviting for participation only people with recorded high levels of dominance and influence, and in parallel initiate discussions including participants with high levels of steadiness and compliance (non-leaders). It should be interesting to examine and analyze the content of such conversations, how and if they will progress, and if it could be easy to conclude to assumptions. Another way to manipulate conversations is the introduction of chatbots, programmed to simulate certain types of personalities. Manipulation will help us recognize in depth how each different personality can maneuver in cultural related conversations. All the aforementioned must be integrated into a set of guidelines for cultural organizations, in order to expand their presence in social media, empower their position in them, try to engage broader audience and spread their message to the public in a more efficient way.

Having already constructed an application that analyzes the outcomes of our research procedure within the scope of the "CrossCult" EU project's pilots, we are able to provide a basic version to cultural institutions, that can perform the experimental procedures and analysis; this will give them the opportunity to participate in serious conversations and have knowledge of the influencers. An online platform, which is able to recognize, analyse and locate discussions based on trending topics, is already constructed, followed by a system that is able to analyze different dimensions of the trending topics in order to identify social media discussions, in which the cultural institutions can participate in order to have higher reach and more relevant audience.

Author Contributions: All the authors contributed equally in this research.

Funding: This work has been partially funded by the project CrossCult: "Empowering reuse of digital cultural heritage in context-aware crosscuts of European history", funded by the European Union's Horizon 2020 research and innovation program, Grant \#693150.

Conflicts of Interest: The authors declare no conflict of interest.

\section{References}

1. Falk, J.H.; Dierking, L.D. The Museum Experience Revisited; Left Coast Press: Walnut Creek, CA, USA, 2013; 416p; ISBN 978-1-61132-044-2.

2. Maslow, A.H. A theory of human motivation. Psychol. Rev. 1943, 50, 370-396. [CrossRef]

3. Duncan, S.; Barrett, L.F. Affect is a form of cognition: A neurobiological analysis. Cognit. Emot. 2007, 21, 1184-1211. [CrossRef] [PubMed]

4. Harmon-Jones, E.; Gable, P.A.; Price, T.F. Does negative affect always narrow and positive affect always broaden the mind? Considering the influence of motivational intensity on cognitive scope. Curr. Direct. Psychol. Sci. 2013, 22, 301-307. [CrossRef]

5. Harmon-Jones, E.; Harmon-Jones, C.; Amodio, D.M.; Gable, P.A. Attitudes toward emotions. J. Pers. Soc. Psychol. 2011, 101, 1332. [CrossRef] [PubMed]

6. Reber, A.S. The Penguin Dictionary of Psychology; Penguin Press: London, UK, 1995.

7. Sheng, C.W.; Chen, M.C. A study of experience expectations of museum visitors. Tour. Manag. 2012, 33, 53-60. [CrossRef]

8. Economou, M.; Meintani, E. Promising Beginning? Evaluating Museum Mobile Phone Apps; Emerging Experiences, University of Limerick: Limerick, Ireland, 2011. 
9. Kuflik, T.; Stock, O.; Zancanaro, M.; Gorfinkel, A.; Jbara, S.; Kats, S.; Kashtan, N. A visitor's guide in an active museum: Presentations, communications, and reflection. J. Comput. Cult. Herit. (JOCCH) 2011, 3, 11. [CrossRef]

10. Gnambs, T.; Batinic, B. A Personality-Competence Model of Opinion Leadership. Psychol. Mark. 2012, 29, 606-621. [CrossRef]

11. Kietzmann, J.H.; Hermkens, K.; McCarthy, I.P.; Silvestre, B.S. Social media? Get serious! Understanding the functional building blocks of social media. Bus. Horiz. 2011, 54, 241-251. [CrossRef]

12. Panahi, S.; Watson, J.; Partridge, H. Social media and tacit knowledge sharing: Developing a conceptual model. World Acad. Sci. Eng. Technol. 2012, 64, 1095-1102.

13. Morris, M.R.; Teevan, J.; Panovich, K. What do people ask their social networks, and why? A survey study of status message q\&a behavior. In Proceedings of the SIGCHI Conference on Human Factors in Computing Systems, Vancouver, BC, Canada, 13-18 April 1996; pp. 1739-1748.

14. Trusov, M.; Bodapati, A.V.; Bucklin, R.E. Determining influential users in internet social networks. J. Mark. Res. 2010, 47, 643-658. [CrossRef]

15. Freberg, K.; Graham, K.; McGaughey, K.; Freberg, L.A. Who are the social media influencers? A study of public perceptions of personality. Public Relat. Rev. 2011, 37, 90-92. [CrossRef]

16. Bakshy, E.; Hofman, J.M.; Mason, W.A.; Watts, D.J. Everyone's an influencer: Quantifying influence on twitter. In Proceedings of the Fourth ACM International Conference on Web Search and Data Mining, Hong Kong, China, 9-12 February 2011; pp. 65-74.

17. Peng, W.; Sun, T. U.S. Patent Method and System for Identifying a Key Influencer in Social Media Utilizing Topic Modeling and Social Diffusion Analysis; No. 8,312,056; U.S. Patent and Trademark Office: Washington, DC, USA, 2012.

18. Gillin, P. The New Influencers: A Marketer's Guide to the New Social Media; Linden Publishing: Fresno, CA, USA, 2009.

19. Booth, N.; Matic, J.A. Mapping and leveraging influencers in social media to shape corporate brand perceptions. Corp. Commun. Int. J. 2011, 16, 184-191. [CrossRef]

20. Parsons, T.; Ratcliffe, M.; Crumpler, R.; Kessler, W.; Freytag, K. U.S. Patent Social Analytics System and Method for Analyzing Conversations in Social Media; No. 8,682,723; U.S. Patent and Trademark Office: Washington, DC, USA, 2014.

21. Sheinman, V.; Fellbaum, C.; Julien, I.; Schulam, P.; Tokunaga, T. Large, huge or gigantic? Identifying and encoding intensity relations among adjectives in WordNet. Lang. Resour. Eval. 2013, 47, 797. [CrossRef]

22. Raison, K.; Tomuro, N.; Lytinen, S.; Zagal, J.P. Extraction of User Opinions by Adjective-Context Co-clustering for Game Review Texts. In Advances in Natural Language Processing; JapTAL 2012. Lecture Notes in Computer Science; Isahara, H., Kanzaki, K., Eds.; Springer: Berlin/Heidelberg, Germany, 2012; Volume 7614.

23. Bouillon, P.; Viegas, E. The description of adjectives for natural language processing: Theoretical and applied perspectives. In Proceedings of the Description des Adjectifs pour les Traitements Informatiques. Traitement Automatique des Langues Naturelles, Cargèse, France, 12-17 July 1999.

24. Fan, W.; Gordon, M.D. The power of social media analytics. Commun. ACM 2014, 57, 74-81. [CrossRef]

25. Colbert, F.; Courchesne, A. Critical issues in the marketing of cultural goods: The decisive influence of cultural transmission. City Cult. Soc. 2012, 3, 275-280. [CrossRef]

26. Scullard, M.; Baum, D. Everything DiSC Manual; Wiley: Hoboken, NJ, USA, 2015.

27. Sugerman, J. Using the DiSC ${ }^{\circledR}$ model to improve communication effectiveness. Ind. Commer. Train. 2009, 41, 151-154. [CrossRef]

28. Krueger, D. Characteristics of the female entrepreneur. J. Bus. Entrep. 2000, 12, 87.

29. Lykourentzou, I.; Naudet, Y.; Vandenabeele, L. Reflecting on European history with the help of technology: The CrossCult project. In Proceedings of the 14th Eurographics Workshop on Graphics and Cultural Heritage, Genova, Italy, 5-7 October 2016; pp. 67-70.

30. Lykourentzou, I.; Antoniou, A.; Naudet, Y.; Dow, S.P. Personality matters: Balancing for personality types leads to better outcomes for crowd teams. In Proceedings of the 19th ACM Conference on Computer-Supported Cooperative Work \& Social Computing, San Francisco, CA, USA, 27 February-2 March 2016; pp. 260-273.

31. Swani, K.; Milne, G.; Brown, B.P. Spreading the word through likes on Facebook: Evaluating the message strategy effectiveness of Fortune 500 companies. J. Res. Interact. Mark. 2013, 7, 269-294. [CrossRef] 
32. Barbieri, F.; Ronzano, F.; Saggion, H. What does this Emoji Mean? A Vector Space Skip-Gram Model for Twitter Emojis. In Proceedings of the 2016 International Conference on Language Resources and Evaluation (LREC), Portoroz, Slovenia, 23-28 May 2016.

33. De Vries, L.; Gensler, S.; Leeflang, P.S. Popularity of brand posts on brand fan pages: An investigation of the effects of social media marketing. J. Interact. Mark. 2012, 26, 83-91. [CrossRef]

34. Mishne, G.; Glance, N. Leave a reply: An analysis of weblog comments. In Proceedings of the Third Annual Workshop on the Weblogging Ecosystem, Edinburgh, UK, 22-26 May 2006.

35. Manago, A.M.; Taylor, T.; Greenfield, P.M. Me and my 400 friends: The anatomy of college students' Facebook networks, their communication patterns, and well-being. Dev. Psychol. 2012, 48, 369. [CrossRef] [PubMed]

36. Ellison, N.B.; Vitak, J.; Gray, R.; Lampe, C. Cultivating social resources on social network sites: Facebook relationship maintenance behaviors and their role in social capital processes. J. Comput. Med. Commun. 2014, 19, 855-870. [CrossRef]

37. Tong, S.T.; Van Der Heide, B.; Langwell, L.; Walther, J.B. Too much of a good thing? The relationship between number of friends and interpersonal impressions on Facebook. J. Comput. Med. Commun. 2008, 13, 531-549. [CrossRef]

38. Bampatzia, S.; Antoniou, A.; Lepouras, G.; Vasilakis, C.; Wallace, M. Using Social media to stimulate history reflection in cultural heritage. In Proceedings of the 2016 11th International Workshop on Semantic and Social Media Adaptation and Personalization (SMAP), Thessaloniki, Greece, 20-21 October 2016; pp. 89-92.

39. Vassilakis, C.; Antoniou, A.; Lepouras, G.; Wallace, M.; Lykourentzou, I.; Naudet, Y. Interconnecting objects, visitors, sites and (Hi) stories across cultural and historical concepts: The CrossCult project. In Proceedings of the Euro-Mediterranean Conference, Nicosia, Cyprus, 31 October-5 November 2016; Springer: Cham, Switzerland, 2016; pp. 501-510.

40. Vassilakis, C.; Antoniou, A.; Lepouras, G.; Poulopoulos, V.; Wallace, M.; Bampatzia, S.; Bourlakos, I. Stimulation of reflection and discussion in museum visits through the use of social media. Soc. Netw. Anal. Min. 2017, 7, 40. [CrossRef]

41. Soren, B.J. Museum experiences that change visitors. Mus. Manag. Curatorship 2009, 24, 233-251. [CrossRef]

42. Bloom, B.S.; Engelhart, M.D.; Furst, E.J.; Hill, W.H.; Krathwohl, D.R. Taxonomy of Educational Objectives, Handbook I: The Cognitive Domain; David McKay Co Inc.: New York, NY, USA, 1956; Volume 19, p. 56.

43. Anderson, L.W.; Krathwohl, D.R.; Airasian, P.; Cruikshank, K.; Mayer, R.; Pintrich, P.; Wittrock, M. A Taxonomy for Learning, Teaching and Assessing: A Revision of Bloom's Taxonomy; Longman Publishing: New York, NY, USA, 2001.

44. Daudelin, M.W. Learning from experience through reflection. Organ. Dyn. 1997, 24, 36-48. [CrossRef]

45. Bakshy, E.; Eckles, D.; Yan, R.; Rosenn, I. Social influence in social advertising: Evidence from field experiments. In Proceedings of the 13th ACM Conference on Electronic Commerce, Valencia, Spain, 4-8 June 2012; pp. 146-161.

46. John, O.P.; Srivastava, S. The Big Five trait taxonomy: History, measurement, and theoretical perspectives. In Handbook of Personality Theory and Research; Pervin, L.A., John, O.P., Eds.; Guilford: New York, NY, USA, 1999; pp. 102-138.

(C) 2018 by the authors. Licensee MDPI, Basel, Switzerland. This article is an open access article distributed under the terms and conditions of the Creative Commons Attribution (CC BY) license (http:/ / creativecommons.org/licenses/by/4.0/). 